\title{
Uso do procedimento de Constructed Response Matching to Sample: uma revisão da literatura
}

\section{Use of Constructed Response Matching to Sample procedure: a literature review \\ El uso de Constructed Response Matching to Sample: una revisión de literatura}

\author{
Glenda Miranda da Paixão', Grauben José Alves de Assis²
} [1] [2] Universidade Federal do Pará I Título abreviado: Uso do procedimento de CRMTS: uma revisão da literatura I Endereço para correspondência: Núcleo de
Teoria e Pesquisa do Comportamento. Rua Augusto Correa, S/n, Guamá. Belém - PA. CEP: 66075-110 I Email: gle_miranda@hotmail.com (Glenda) ggrauben@
gmail.com (Grauben) I DOI: 10.18761/PAC.2016.038

Resumo: Realizou-se pesquisa nos periódicos nacionais e internacionais a fim de analisar as publicações que envolvem o procedimento de Constructed Response Matching to Sample (CRMTS), identificando as variáveis de procedimento facilitadoras do aprendizado de repertórios de leitura e escrita. Os termos de busca utilizados estavam em língua portuguesa (anagrama, construção e cópia) ou em língua inglesa (Constructed response matching to sample, anagram, constructed, construction e spelling). A sigla CRMTS também foi utilizada como termo de busca. Foram encontrados 571 artigos, sendo 35 selecionados para análise. O procedimento de CRMTS tem se mostrado eficiente para demonstrar a emergência de relações de equivalência. A informatização do procedimento, o uso das unidades verbais mínimas e procedimentos de correção combinados se mostraram eficientes na geração de aprendizagem, porém os resultados acerca da generatividade têm mostrado variabilidade. As variáveis envolvidas na propriedade generativa precisam ser melhor investigadas, bem como a generalização dos dados obtidos em ambiente controlado para ambientes naturais de aprendizagem.

Palavras-chave: CRMTS, spelling, discriminação condicional, equivalência de estímulos. 
Abstract: Research was carried out in national and international journals in order to analyze the publications involving the Constructed Response Matching to Sample (CRMTS) procedure, identifying variables that facilitate the learning of reading and writing repertoires. The search terms used were in Portuguese (anagrama, construção and cópia) or English (Constructed response matching to sample, anagram, constructed, construction and spelling). The CRMTS abbreviation was also used as a search term. Were found 571 articles, 35 were selected for analysis. The CRMTS procedure has been shown to be efficient for demonstrating the emergence of equivalence relations. The combined use of computerized procedure, minimal verbal units and procedures for correcting showed efficiency in generating learning, however the results on the generativity have shown variability. The variables involved in the generative property need to be better investigated, as well as the generalization of data obtained in a controlled environment for natural environments of learning.

Keywords: CRMTS, spelling, condicional discrimination, stimulus equivalence..

Resumen: Se llevó a cabo la investigación en revistas nacionales e internacionales con el fin de analizar las publicaciones relativos a procedimiento de Constructed Response Matching to Sample (CRMTS), com la identificación de las variables de procedimiento de facilitación de aprendizaje de repertorios de lectura y escritura. Los términos de búsqueda utilizados fueron en portugués (anagrama, construção y copia) o Inglés (Constructed response matching to sample, anagram, constructed, construction and spelling). El acrónimo CRMTS también fue utilizado como un término de búsqueda. Fueran encontrados 571 artículos, 35 fueran seleccionados para el análisis. El procedimiento CRMTS ha demostrado ser eficaz para demostrar la aparición de relaciones de equivalencia. La informatización del procedimiento, el uso de unidades verbales mínimas y la corrección de los procedimientos combinados fueran eficientes en la generación de aprendizaje, pero los resultados sobre la generatividad han mostrado variabilidad. Las variables que intervienen en la propiedad generativa necesitan más investigación así como la generalización de los datos obtenidos en ambiente controlado para los entornos naturales de aprendizaje.

Palabras-clave: CRMTS, spelling, discriminación condicional, equivalencia de estímulos. 
A aprendizagem, segundo Goulart, Delage, Rico e Brino (2012), é "qualquer mudança duradoura na maneira como os organismos respondem ao ambiente" (p. 21). Essa mudança pode ser tanto a modificação de uma relação estímulo-resposta preexistente quanto o estabelecimento de uma nova relação. Dois processos de aprendizagem básicos são descritos na literatura da análise do comportamento: o condicionamento respondente e o condicionamento operante, sendo este último definido como uma correlação entre um estímulo reforçador e uma resposta, quando esta correlação produz modificações na probabilidade de que certa resposta ocorra (Goulart et al., 2012; Skinner, 1938).

O processo de condicionamento operante pelo qual um organismo aprende a responder diferencialmente na presença ou na ausência de um estímulo antecedente é descrito como discriminação. Existem dois tipos básicos de discriminação: a simples e a condicional. Entende-se como discriminação simples as situações em que um comportamento é evocado por um mesmo agrupamento de propriedades de estímulos. Em uma situação de discriminação condicional não se estabelece uma relação constante entre um estímulo antecedente e uma resposta, esta relação muda de acordo com os contextos nos quais este estímulo aparece, ou seja, uma determinada resposta é reforçada na presença de um estímulo específico apenas se um outro estímulo estiver presente (Debert, Matos, \& Andery, 2006). Entre as décadas de 1950 e 1960 os estudos em análise do comportamento apresentaram um grande desenvolvimento de pesquisas envolvendo discriminações condicionais, devido à introdução do procedimento de emparelhamento de acordo com o modelo (MTS - matching-to-sample), composto por estímulos condicionais apresentados como estímulos modelo, e estímulos de discriminação apresentados como estímulos de escolha. Respostas corretas são seguidas de reforço e intervalo entre tentativas, e respostas incorretas são seguidas de intervalo entre tentativas. Neste arranjo, é possível apresentar o modelo e as escolhas simultaneamente ou não e ainda, o estímulo modelo pode ser idêntico a um dos estímulos de escolhas ( $m a-$ tching de identidade) ou não (matching arbitrário) (Matos, 1999).

Um conjunto de estudos utilizando o procedimento de MTS foi conduzido ao longo da década de 1970, usando o paradigma de equivalência de estímulos (Sidman \& Tailby, 1982). Segundo esse paradigma, quando uma relação é explicitamente ensinada, outras relações não diretamente ensinadas podem emergir ou pelo menos serem fortalecidas (de Rose, 1996). Para isso, procedimentos de ensino têm sido usados pelos analistas do comportamento para estudar a formação de classes de equivalência que podem constituir uma ferramenta metodológica relevante para estudar o comportamento simbólico. A formação de classes de equivalência e o comportamento simbólico têm em comum o fato de elementos sem similaridade física, uma vez arbitrariamente relacionados, se tornarem equivalentes, ou seja, substituíveis no que diz respeito ao controle de repertórios a eles associados (Barros, Galvão, Brino, Goulart, \& Mcllvane, 2005).

Uma variação do procedimento de MTS tem sido descrita na literatura por Constructed Response Matching to Sample (CRMTS), utilizado inicialmente no ensino de spelling (ordenação de letras em uma sequência). Nas tarefas de CRMTS, a comparação correta é construída pelo participante por meio da seleção de seus componentes individuais (de Souza \& de Rose, 2006; Dube, McDonald, Mcllvane, \& Mackay, 1991). As escolhas podem estar relacionadas ao modelo com base nas características físicas comuns ou com base em relações arbitrárias.

Mackay e Sidman (1984) organizaram um procedimento de construção de palavras com um estudante com deficiência intelectual. O estudante já relacionava nomes ditados às cores e já nomeava as cores. O modelo era composto (cartão colorido e palavra impressa referente ao nome da cor) e o estudante deveria apenas "cobrir" o modelo presente num cartão com letras móveis disponíveis, na ordem da esquerda para a direita. Em seguida, os experimentadores ensinaram-no a construir o nome das cores na presença de modelos ditados. Foi verificado que o participante, apresentou construção da palavra impressa diante do modelo ditado e na presença da cor. Além disso, o estudante relacionou condicionalmente as palavras ditadas às palavras impressas, as cores às palavras impressas e vice-versa, e apresentou leitura textual das palavras impressas, demonstrando a formação de uma classe de estímulos equivalentes. Outros três estu- 
dantes com deficiência intelectual foram ensinados a construir a palavra impressa na presença da cor e da palavra impressa, porém com remoção das letras da palavra-modelo, resultando no desempenho de construção da palavra diante somente da cor.

A partir de então, diferentes arranjos experimentais do procedimento de CRMTS foram descritos na literatura. O objetivo do presente estudo foi apresentar uma revisão da literatura da análise do comportamento acerca do uso desse procedimento, classificando com quais populações tem sido aplicado, analisando os delineamentos experimentais e os procedimentos metodológicos adotados e os efeitos destes nos principais resultados descritos, visando identificar as variáveis que parecem favorecedoras do aprendizado de repertório de leitura e de escrita (construção).

\section{Método}

Inicialmente, foi realizado um levantamento no portal de periódicos da Capes, com o termo Constructed Response Matching-to-Sample, nas bases de dados Scielo e Medline, sendo encontrados 62 artigos, porém apenas 16 versavam sobre o tema abordado. Consultou-se, então, nove periódicos nacionais e 14 internacionais apontados pelo levantamento anterior, totalizando 23 periódicos. Os termos de busca utilizados estavam em língua portuguesa (anagrama, construção e cópia) ou em língua inglesa (Constructed response matching to sample, anagram, constructed, construction e spelling). O termo de busca CRMTS também foi utilizado. Nenhuma limitação de data foi definida.

Os artigos selecionados deveriam citar, no resumo, um procedimento de construção de respostas. Quando este procedimento não estava claro no resumo, era acessado a seção de método, na qual um procedimento de CRMTS deveria estar presente. Foram excluídos os artigos que: a) não apresentavam relação com o tema abordado, b) estavam fora do escopo da análise do comportamento, c) não apresentavam procedimento de CRMTS em nenhuma fase descrita na sessão de método, d) as revisões bibliográficas e, e) artigos repetidos.

\section{Resultados e Discussão}

Os termos de busca identificaram 571 artigos, 296 nos periódicos nacionais e 275 nos periódicos internacionais. A partir dos títulos e dos resumos, excluíram-se os textos que não apresentavam relação com o tema abordado e/ou estavam fora do escopo da análise do comportamento. Dos 53 artigos pré-selecionados (40 de periódicos internacionais e treze de periódicos nacionais), após leitura do resumo ou da sessão de método, foram excluídos aqueles que não apresentavam procedimento de CRMTS descrito na sessão de método, revisões bibliográficas e artigos repetidos. Trinta e cinco artigos foram, então, selecionados para a análise, dez em periódicos nacionais e 25 em periódicos internacionais. Os termos de busca que mais geraram artigos selecionados para análise foram CRMTS, nos periódicos nacionais, com oito artigos, e spelling, nos periódicos internacionais, com 18 artigos selecionados.

O período de maior publicação nos periódicos internacionais foi o de 2006 a 2010, com dez publicações registradas, e nos periódicos nacionais, o período de 2011 até o momento, tendo sido encontradas oito publicações (ver Figura 1).

Trinta e três artigos objetivaram demonstrar relações de equivalência entre estímulos, 31 deles a partir do ensino de repertório de leitura textual e leitura com compreensão, bem como a construção (ou escrita) de palavras e, em dois deles, a partir do ensino de comportamentos matemáticos. O ensino desses repertórios é fundamental, uma vez que, segundo Greer (2002), o comportamento de ler fluentemente, e outros comportamentos relacionados à leitura são necessários ao sucesso individual e à contribuição social relevante no presente milênio. Portanto, esclarecer as variáveis envolvidas no processo de ensino-aprendizagem de leitura e escrita e demonstrar procedimentos eficientes e eficazes para o ensino desses repertórios tornou-se um objetivo importante na literatura da análise do comportamento.

Vinte e cinco artigos (ver Tabela 1) envolveram o ensino da construção das palavras, utilizando as palavras como modelo (impressa ou ditada, ou ainda, uma figura representativa) e a 


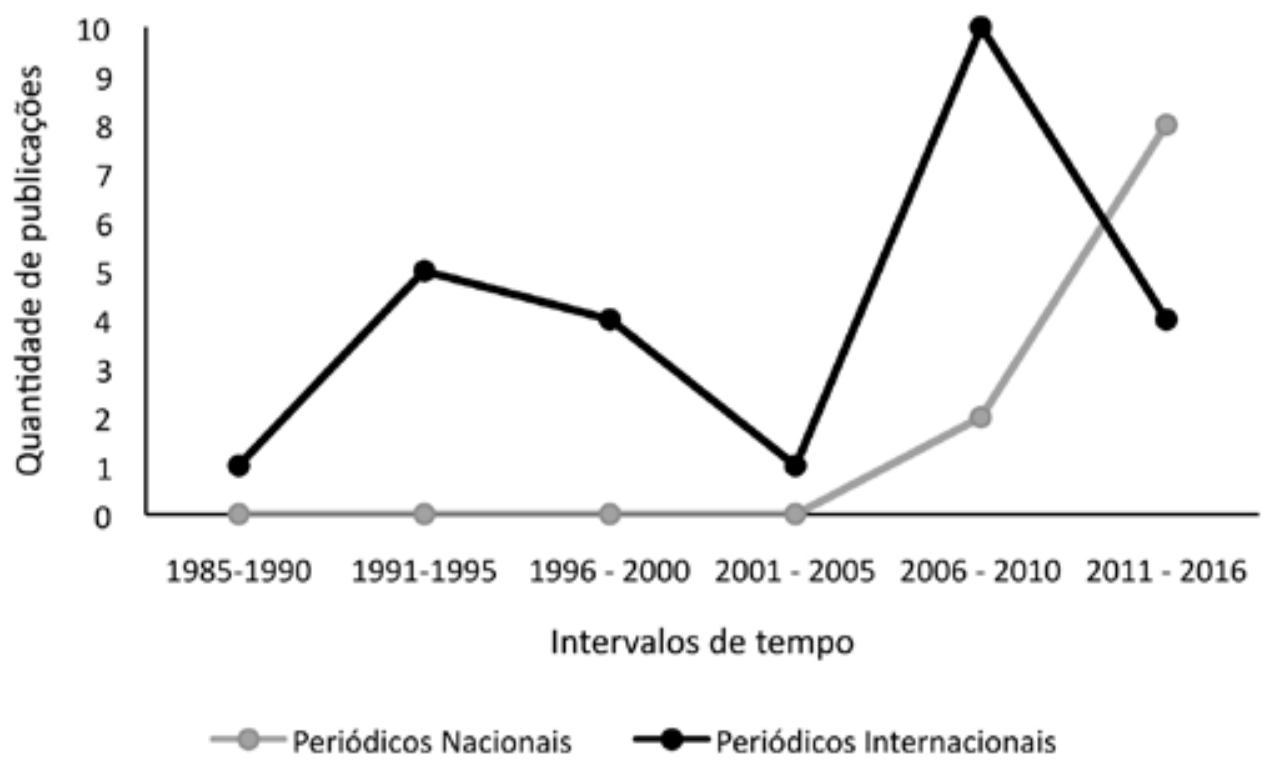

Figura 1 - Quantidade de artigos publicados em periódicos nacionais e internacionais no decorrer dos anos, de 1985 até o momento.

letra ou sílaba como estímulos de comparação. O ensino por unidades verbais mínimas (Skinner, 1992) tem sido apontado como mais eficiente em gerar repertórios novos, principalmente quando tais unidades são sílabas e letras. Observa-se que quatro estudos envolvendo o ensino de construção de palavras em língua portuguesa utilizaram a sílaba como unidade mínima (Domeniconi, de Rose \& Huziwara, 2007; Sella, Tenório, Bandini \& Bandini, 2016; Sudo, Soares, de Souza \& Haydu, 2008; Zanco \& Moroz, 2015), embora dois deles (Sella, Tenório, Bandini e Bandini, 2016; Zanco e Moroz, 2015) também tenham reportado etapas de construção pela seleção de letras.

Seis estudos envolveram tarefas de ensino de construção de sentenças (impressas, ditadas ou sinais em LIBRAS) pela seleção de palavras (ver Tabela 1). Em cinco desses estudos (Assis, Calado, \& de Souza, 2016; Fonseca, Assis, \& de Souza, 2015; Haydu, Zuanazzi, Assis, \& Kato, 2015; Yamamoto \& Miya, 1999), os participantes já apresentavam leitura de palavras em seu repertório de entrada e, ainda assim, em dois estudos (Fonseca, Assis, \& de Souza, 2015; Haydu, Zuanazzi, Assis, \& Kato,
2015) foram desenvolvidas estratégias para fortalecimento desses repertórios para posterior ensino de sentenças. Em um último estudo (Santos, Assis, \& Borba, 2016), o ensino de leitura de palavras teve que ser ensinado em etapas prévias ao ensino de sentenças. Esses procedimentos demonstram a importância do controle pelas unidades mínimas para o desenvolvimento de repertórios de construção de sentenças.

Dois estudos abordaram o ensino de comportamentos matemáticos (conceito de proporção e valores monetários). O comportamento matemático é uma subdivisão do comportamento verbal que apresenta um vocabulário aritmético, uma sintaxe, uma estrutura de equações e outros tipos de funções, e de encadeamento, que ocorre tanto na comunicação, como no pensamento (Rossit \& Ferreira, 2003). Para os estudos de Magalhães e Assis (2011) e Santos, Simonassi, Rodrigues e Magri (2014), o CRMTS não mostrou efeitos sobre o aprendizado dos repertórios selecionados pelos experimentadores. Assim, não existe nas publicações avaliadas, evidências empíricas relevantes do uso do CRMTS para o ensino de conceitos matemáticos. 
Tabela 1. Estímulos utilizados como modelo e como comparação.

\begin{tabular}{|c|c|c|}
\hline Referência & Estímulo modelo & Estímulos de comparação \\
\hline Mackay (1985) & Palavras & Letras \\
\hline Dube, Mcdonald, Mcllvane e Mackay, (1991) & Palavras & Letras \\
\hline Stromer e Mackay (1992a) & Palavras & Letras \\
\hline Strommer e Mackay (1992b) & Palavras & Letras \\
\hline Stromer e Mackay (1993) & Palavras & Letras \\
\hline Stromer Mackay, Howell, McVay e Flusser (1996) & Palavras & Letras \\
\hline de Rose, de Souza e Hanna (1996) & Palavras & Letras \\
\hline Yamamoto e Myia (1999) & Sentenças & Palavras (em Kanji) \\
\hline Hanna, de Souza, de Rose e Fonseca (2004) & Palavras & Letras \\
\hline Lee-Vieira, Mayer e Cameron (2006) & Palavras & Letras \\
\hline Matos, Avanzi e Mcllvane (2006) & Palavras & Sílabas \\
\hline Vedora e Stromer (2007) & Palavras & Letras \\
\hline Sugasawara e Yamamoto (2007) & Palavras & Sílabas (em Hiragana) \\
\hline de Souza, Goyos, Silvares e Saunders (2007) & Palavras & Letras \\
\hline Domeniconi, de Rose e Huziwara, (2007) & Palavra & Sílaba \\
\hline Sudo, Soares, de Souza e Haydu (2008) & Palavra & Sílaba \\
\hline Mayfield, Glenn e Vollmer (2008) & Palavras & Letras \\
\hline Sugasawara e Yamamoto (2009) & Palavras (em Kanji) & Sílabas (em Kanji) \\
\hline $\begin{array}{l}\text { de Souza de Rose, Faleiros, Bortoloti, Hanna e Mcllvane } \\
\text { (2009) }\end{array}$ & $\begin{array}{l}\text { Sílabas } \\
\text { Palavras }\end{array}$ & $\begin{array}{l}\text { Letras } \\
\text { Sílabas }\end{array}$ \\
\hline de Souza e Hubner (2010) & Palavras & Sílabas \\
\hline Tanji e Noro (2011) & Palavras em Hiragana & Sílabas em Hiragana \\
\hline Resende, Elias e Goyos (2012) & Sentenças & Palavras \\
\hline Reis, Postalli e de Souza (2013) & Palavras & Letras \\
\hline Mackay (2013) & $\begin{array}{l}\text { Palavras } \\
\text { Números }\end{array}$ & Letras \\
\hline Fonseca, Assis e de Souza (2015) & Sentenças & Palavras \\
\hline Zanco e Moroz (2015) & Palavras & Letras e sílabas \\
\hline Haydu, Zuanazzi, Assis e Kato (2015) & Sentenças & Palavras \\
\hline Santos, Assis e Borba (2016) & Sentenças & Palavras \\
\hline Assis, Calado e de Souza (2016) & Sentenças & Palavras \\
\hline Sella, Tenório, Bandini e Bandini (2016) & Palavras & Sílabas e letras \\
\hline Benitez e Domeniconi (2016) & Palavras & Letras e Sílabas \\
\hline
\end{tabular}

Quanto aos participantes (ver Tabela 2), houve doze registros de escolares típicos, e 23 estudos reportaram participação de populações especiais, como surdos (Magalhães \& Assis, 2011; Santos, Assis, \& Borba, 2016; Resende, Elias, \& Goyos, 2012), pessoas com autismo (Calcagno, Dube, Galvão, \&

Sidman, 1994; Stromer, Mackay, Howell, McVay, \& Flusser, 1996; Tanji \& Noro, 2011; Yamamoto \& Miya, 1999), déficit cognitivo (Benitez \& Domeniconi, 2016; Calcagno, Dube, Galvão, \& Sidman, 1994; Dube, Mcdonald, McIlvane, \& Mackay, 1991, Stromer \& Mackay, 1992a; 
1992b; 1993; Stromer, Mackay, Howell, McVay, \& Flusser,1996), dificuldade de aprendizagem (De Souza, Goyos, Silvares, \& Sanders, 2007; Hanna, de Souza, de Rose, \& Fonseca, 2004; Lee-Vieira, Mayer, \& Cameron, 2006; Sugasawara \& Yamamoto, 2007;
Reis, Postalli, \& de Souza, 2013; Zanco \& Moroz, 2015), síndrome de Down (Domeniconi, de Rose, \& Huziwara, 2007; Mackay, 1985), atraso no desenvolvimento (Sugasawara \& Yamamoto, 2007) e paralisia cerebral (Mackay, 2013).

\section{Tabela 2. Características dos participantes.}

\begin{tabular}{|c|c|c|c|c|}
\hline Referência & Participantes & Idades & Número & Delineamento \\
\hline Mackay (1985) & Pessoas com síndrome de Down. & Sem informação & 3 & Sujeito único \\
\hline $\begin{array}{l}\text { Dube, Mcdonald, Mcllvane e } \\
\text { Mackay (1991) }\end{array}$ & Pessoas com déficit cognitivo & 24 e 27 anos & 2 & Sujeito único \\
\hline Stromer \& Mackay (1992a) & Pessoas com déficit cognitivo. & $\begin{array}{l}\text { Entre nove e treze } \\
\text { anos }\end{array}$ & 3 & Sujeito único \\
\hline Strommer e Mackay (1992b) & Pessoas com déficit cognitivo. & $\begin{array}{l}\text { Não disponibiliza } \\
\text { idade }\end{array}$ & 3 & Sujeito único \\
\hline Stromer e Mackay (1993) & Pessoas com déficit cognitivo & 14 e 21 anos & 2 & Sujeito único \\
\hline $\begin{array}{l}\text { Calcagno, Dube, Galvão e } \\
\text { Sidman, (1994) }\end{array}$ & $\begin{array}{l}\text { Pessoas com déficit cognitivo, uma } \\
\text { delas com diagnóstico de TEA }\end{array}$ & $\begin{array}{l}\text { Idades entre } 13 \text { e } \\
21 \text { anos }\end{array}$ & 3 & Sujeito único \\
\hline $\begin{array}{l}\text { Stromer Mackay, Howell, McVay e } \\
\text { Flusser (1996) }\end{array}$ & $\begin{array}{l}\text { Pessoas com déficit cognitivo e auditi- } \\
\text { vo, uma delas diagnosticada com } \\
\text { autismo. }\end{array}$ & 21 e 40 anos & 2 & Sujeito único \\
\hline de Rose, de Souza, Hanna (1996) & Escolares típicos & $\begin{array}{l}\text { Entre sete e dez } \\
\text { anos }\end{array}$ & 11 & Grupo \\
\hline Birnie-Selwyn e Guerin (1997) & Escolares típicos & Entre 4 e 7 anos & 6 & Sujeito único \\
\hline Yamamoto e Myia (1999) & Pessoas com diagnóstico de TEA & Entre 6 e 10 anos & 3 & Sujeito único \\
\hline $\begin{array}{l}\text { Hanna, de Souza, de Rose e } \\
\text { Fonseca (2004) }\end{array}$ & $\begin{array}{l}\text { Escolares com história de dificuldade } \\
\text { de aprendizagem. }\end{array}$ & Entre 8 e 10 anos & 6 & Sujeito único \\
\hline $\begin{array}{l}\text { Lee-Vieira, Mayer e Cameron } \\
\text { (2006) }\end{array}$ & $\begin{array}{l}\text { Escolares com história de dificuldade } \\
\text { de aprendizagem. }\end{array}$ & $\begin{array}{l}\text { Não disponibiliza } \\
\text { idade }\end{array}$ & 10 & Sujeito único \\
\hline Matos, Avanzi e Mcllvane (2006) & Escolares de famílias de baixa renda & Entre 5 e 6 anos & 16 & Sujeito único \\
\hline Vedora e Stromer (2007) & Pessoas com déficit cognitivo & 14 e 17 anos & 2 & Sujeito único \\
\hline Sugasawara e Yamamoto (2007) & $\begin{array}{l}\text { Escolares com atraso no } \\
\text { desenvolvimento }\end{array}$ & 4 e 12 anos & 2 & Sujeito único \\
\hline $\begin{array}{l}\text { de Souza, Goyos, Silvares e } \\
\text { Saunders (2007) }\end{array}$ & $\begin{array}{l}\text { Pré-escolares típicos e escolares com } \\
\text { dificuldade na escrita. }\end{array}$ & Entre 3 e 10 anos & 9 & Sujeito único \\
\hline $\begin{array}{l}\text { Domeniconi, de Rose e Huziwara } \\
\text { (2007) }\end{array}$ & Pessoas com Síndrome de Down. & Entre 7 e 24 anos & 4 & Sujeito único \\
\hline $\begin{array}{l}\text { Sudo, Soares, de Souza e Haydu } \\
\text { (2008) }\end{array}$ & Escolares típicos & Entre 6 e 8 anos & 3 & Sujeito único \\
\hline Mayfield, Glenn e Vollmer (2008) & Escolares típicos. & 12 anos & 2 & Sujeito único \\
\hline Sugasawara e Yamamoto (2009) & Escolares com dificuldade na escrita & 8 anos & 1 & Sujeito único \\
\hline $\begin{array}{l}\text { de Souza, de Rose, Faleiros, } \\
\text { Bortoloti, Hanna e Mcllvane } \\
\text { (2009) }\end{array}$ & Escolares típicos & $\begin{array}{l}\text { E1: entre } 8 \text { e } 12 \\
\text { anos }\end{array}$ & $\begin{array}{l}\text { E1: } 12 \\
\text { E2: } 17\end{array}$ & Grupo \\
\hline de Souza e Hubner (2010) & Escolares típicos & Entre 5 e 6 anos. & 9 & Grupo \\
\hline
\end{tabular}




\begin{tabular}{|c|c|c|c|c|}
\hline Referência & Participantes & Idades & Número & Delineamento \\
\hline Tanji e Noro (2011) & $\begin{array}{l}\text { Pessoas com déficit cognitivo, uma } \\
\text { diagnosticada com TEA. }\end{array}$ & 8 e 12 anos & 2 & Sujeito único \\
\hline Magalhães e Assis (2011) & Crianças surdas & Entre 9 e 10 anos. & 10 & Grupo \\
\hline Resende, Elias e Goyos (2012) & Pessoas surdas. & Entre 8 e 16 anos & 4 & Sujeito único \\
\hline Reis, Postalli e Souza (2013) & $\begin{array}{l}\text { Escolares com dificuldade de apren- } \\
\text { dizagem }\end{array}$ & Entre 6 e 8 anos & 4 & Sujeito único \\
\hline Mackay (2013) & Criança com Paralisia Cerebral. & 10 anos & 1 & Sujeito único \\
\hline $\begin{array}{l}\text { Santos, Simonassi, Rodrigues e } \\
\text { Magri (2014) }\end{array}$ & Escolares típicos & $\begin{array}{l}\text { Entre } 11 \text { e } 13 \\
\text { anos }\end{array}$ & 20 & Grupo \\
\hline $\begin{array}{l}\text { Fonseca, Assis e de Souza } \\
(2015)\end{array}$ & Escolares típicos & Entre 6 a 8 anos & 5 & Sujeito único \\
\hline Zanco e Moroz (2015) & $\begin{array}{l}\text { Escolares com dificuldades de apren- } \\
\text { dizagem }\end{array}$ & Sem informação & 4 & Sujeito único \\
\hline $\begin{array}{l}\text { Haydu, Zuanazzi, Assis e Kato } \\
\text { (2015) }\end{array}$ & Escolares típicos & Entre 7 e 9 anos & 8 & Sujeito único \\
\hline Santos, Assis e Borba (2016) & Crianças surdas & Entre 9 e 13 anos. & 3 & Sujeito único \\
\hline Assis, Calado e de Souza, (2016) & Escolares típicos & Entre 6 e 8 anos & 5 & Sujeito único \\
\hline $\begin{array}{l}\text { Sella, Tenório, Bandini e Bandini, } \\
\text { (2016) }\end{array}$ & $\begin{array}{l}\text { Escolares com dificuldades de apren- } \\
\text { dizagem }\end{array}$ & Entre 7 e 12 anos & 4 & Sujeito único \\
\hline Benitez e Domeniconi (2016) & Pessoas com déficit cognitivo. & $\begin{array}{l}\text { Entre } 14 \text { e } 26 \\
\text { anos }\end{array}$ & 5 & Sujeito único \\
\hline
\end{tabular}

Constatou-se que nas pesquisas que envolveram o ensino de habilidades acadêmicas os participantes são justamente aqueles que se beneficiariam da tecnologia de ensino produzida, o que está de acordo com os achados de De Paula e Haydu (2010) em seu levantamento bibliográfico sobre os estudos brasileiros envolvendo equivalência de estímulos. Em relação ao número de participantes, 24 estudos tiveram de um a oito participantes. Oito estudos tiveram de nove a vinte participantes, em cinco deles os participantes foram divididos em grupos. Os números baixos de participantes se devem ao fato do uso do delineamento de sujeito-único nas pesquisas em análise do comportamento. Neste tipo de delineamento, os sujeitos são expostos a uma série de condições e o desempenho de cada indivíduo é mensurado repetidamente, verificando-se se há uma relação ordenada entre as condições manipuladas e as alterações nessas medidas, e as diferenças entre sujeitos não comprometem a análise de dados, pois cada participante tratado como um indivíduo particular (Sampaio et al., 2008; Martone \& Santos-Carvalho, 2012).

Sobre a apresentação do modelo (ver Tabela 3), sete estudos apresentaram o modelo com atraso, nesta modalidade de procedimento, somente após a remoção do modelo é que os estímulos-comparação são apresentados e a tarefa pode ser realizada. A apresentação do modelo com atraso foi apresentada como uma alternativa ao fading, descrito como procedimento adicional em três estudos, definido como alteração gradual nas propriedades de estímulos que definem uma classe operante discriminada (Catania, 1999).

Assis, Fonseca e Bandeira (2014), avaliaram o uso do fading como importante para a transferência de controle do modelo impresso (visual) para o modelo ditado (auditivo). Dube et al. (1991) também concluíram que procedimento de fading permitiu a seleção da letra correta e mesmo após a retirada do procedimento, os escores de acertos permaneceram altos. Diferenças importantes nos efeitos no aprendizado dos sujeitos não foram encontradas entre estudos com CRMTS com atraso e fading. Vinte e quatro estudos apresentaram o modelo de forma simultânea, dois estudos não deixam claro a apresentação do modelo. Um estudo utiliza CRMTS como procedimento de correção e outro utiliza CRMTS apenas nas tarefas de teste. 
Tabela 3. Variações de procedimentos

\begin{tabular}{|l|l|l|l|l|l|l|l|l|}
\hline & \multicolumn{2}{|l|}{ Apresentação do modelo } & $\begin{array}{l}\text { Procedimento } \\
\text { informatizado }\end{array}$ & $\begin{array}{l}\text { Procedimento } \\
\text { adicional }\end{array}$ & Procedimento de correção \\
\hline & Simultâneo & Com atraso & $\operatorname{Sim}$ & Não & MTS & Fading & $\begin{array}{l}\text { Reexposição à } \\
\text { tentativa }\end{array}$ & Outros \\
\hline $\begin{array}{l}\text { Quantidade } \\
\text { de artigos }\end{array}$ & 24 & 7 & 27 & 8 & 24 & 3 & 12 & 6 \\
\hline
\end{tabular}

Procedimentos informatizados foram encontrados em 27 estudos (ver Tabela 3). Esta preferência tem como base o fato de que o uso de computadores exige topografias de respostas motoras mais simples e possibilitam feedback imediato. Além disso, de acordo com Mayfield, Glenn e Vollmer (2008), a informatização permite que as tarefas sejam realizadas sem a supervisão extensiva de um adulto e diminuem o número de reforçadores que devem ser dispensados.

Dezoito estudos descrevem procedimentos de correção (ver Tabela 3), sendo em doze deles a reexposição à tentativa a correção adotada. Outros estudos citam o uso do CRMTS com atraso (Sudo, Soares, de Souza \& Haydu, 2008), apresentação da resposta correta na tela por 5s (Yamamoto \& Miya, 1999), comparação da resposta com o estímulo modelo e revisão (Hanna, de Souza, de Rose, \& Fonseca, 2004; Lee-Vieira, Mayer, \& Cameron, 2006) e uso da tecla Start Over que permitia ao participante começar novamente a tentativa (Vedora \& Stromer, 2007). Estudos acerca dos procedimentos de correção durante ensino de tentativas discretas vêm sendo relatados na literatura da análise do comportamento, apontando diferenças idiossincráticas entre os participantes, o que sugere que os procedimentos de correção deveriam ser individualizados, entretanto, a reapresentação até a correção de erros independente parece levar à aquisição mais rápida habilidade (Carroll, Joachim, St. Petter, \& Robinson, 2015).

Trinta artigos que tratam do aprendizado de leitura e construção de palavras e sentenças apre- sentam o CRMTS nas tarefas de ensino, combinado ou não ao procedimento de MTS. Os resultados desses artigos apontam para a formação de classes de estímulos equivalentes entre os estímulos utilizados no ensino. Destes, 19 artigos também investigaram a generatividade do procedimento (ver Tabela 4), porém doze apresentaram dados parciais com relação às respostas dos participantes a novos estímulos não treinados diretamente. Verificar as variáveis que afetam a propriedade generativa dos procedimentos baseados no paradig ma de equivalência de estímulos, segundo a qual o ensino de algumas relações pode ser seguido pela emergência de múltiplas novas relações (de Rose, 1996) é de extrema importância para a busca de procedimentos de ensino cada vez mais eficientes e econômicos, que auxiliem no ensino e na remediação de repertórios de leitura e escrita, além de outros repertórios acadêmicos.

Estudos também relataram a formação de classes sintáticas, sendo estas um exemplo da relação entre eventos antecedentes e consequentes, pois a disposição das palavras em uma frase segue uma determinada ordem, estabelecida pela comunidade verbal para que a frase tenha sentido (Haydu, Zuanazzi, Assis, \& Kato, 2015). Segundo Assis, Baptista e Nunes (2009), a estrutura sintática de uma sentença indica as relações que as palavras estabelecem umas com as outras para que a sentença tenha sentido e seja compreendida. Nos três estudos que discutiram dados sob a ótica da formação das classes sintáticas, os dados dos testes de generalização mostraram-se parciais. 
Tabela 4. Artigos que investigaram formação de classes de equivalência e generatividade

\begin{tabular}{|c|c|c|}
\hline Referência & Formação de classes & Generatividade \\
\hline de Souza, de Rose, Hanna (1996) & Sim & Sim \\
\hline Yamamoto e Myia (1999) & Sim & Parcial \\
\hline Hanna, de Souza, de Rose e Fonseca (2004) & Sim & Parcial \\
\hline Matos, Avanzi e Mcllvane (2006) & Sim & Sim \\
\hline de Souza, Goyos, Silvares e Saunders (2007) & Sim & Sim \\
\hline Sudo, Soares, de Souza e Haydu (2008) & Sim & Parcial \\
\hline Sugasawara e Yamamoto (2009) & Sim & Sim \\
\hline de Souza, de Rose, Faleiros, Bortoloti, sim Hanna e Mcllvane (2009) & Sim & Parcial \\
\hline De Souza e Hubner (2010) & Sim & Parcial \\
\hline Tanji e Noro (2011) & Sim & Sim \\
\hline Resende, Elias e Goyos (2012) & Sim & Parcial \\
\hline Reis, Postalli e de Souza (2013) & Sim & Parcial \\
\hline Mackay (2013) & Sim & Sim \\
\hline Fonseca, Assis e de Souza (2015) & Sim & Sim \\
\hline Zanco e Moroz (2015) & Sim & Parcial \\
\hline Haydu, Zuanazzi, Assis e Kato (2015) & Parcial & Parcial \\
\hline Santos, Assis e Borba (2016) & Sim & Parcial \\
\hline Assis, Calado e de Souza (2016) & Parcial & Parcial \\
\hline Sella, Tenório, Bandini e Bandini (2016) & Sim & Parcial \\
\hline
\end{tabular}

Com relação às principais discussões, as mesmas se reportaram para a formação de classes de equivalência. Alguns estudos, porém, discutiram a aplicabilidade de procedimentos informatizados realizados em domicílio, num contexto de estudo autodirigido e a aplicação dos procedimentos por mães e por funcionários da escola. Os procedimentos via CRMTS, informatizados ou não, mostraram-se eficientes em contextos naturais de aprendizagem, mesmo com menor controle das variáveis estranhas, replicando seus efeitos em uma escola pública e em domicílios, havendo ainda a demonstração de que tal procedimento pode ser aplicado por pessoas sem extensivo treino nos modelos e métodos da análise do comportamento, mostrando-se uma alternativa viável para o ensino e remediação de repertórios acadêmicos em ambiente natural de aprendizagem dos mesmos.

Avaliando os dados dos estudos apresentados, verifica-se, de uma forma geral, que a utilização do procedimento de CRMTS tem se apresentado como uma possibilidade eficiente no controle de estímulos com diferentes populações. O uso do procedimento informatizado, o ensino das unidades verbais mínimas, a graduação da complexidade da tarefa e a utilização de procedimentos de correção combinados se mostraram eficientes na geração de repertórios novos, na aprendizagem tanto de pessoas com desenvolvimento típico quanto de pessoas com desenvolvimento atípico, demonstrando a eficiência da implementação dessa tecnologia de ensino. Repertórios novos foram observados durante os testes finais nos quais as tarefas exigiam a nomeação ou construção de palavras, sentenças ou textos que não haviam sido apresentadas aos participantes durante as fases de ensino. Além disso, Yamamoto e Miya (1999) e Stromer et al. (1996) testaram também a formação de repertórios novos entre modalidades de respostas, testando os efeitos de procedimentos de ensino informatizados na escrita à mão. A propriedade generativa é importante, pois ao permitir a emergência de relações 
não diretamente ensinadas, pode originar formas econômicas e eficientes de construir ou remediar repertórios relacionais complexos, em ambientes educacionais ou terapêuticos, incluindo estudos envolvendo indivíduos com habilidades linguísticas muito limitadas (de Rose, 1996).

Entretanto, as variáveis capazes de gerar responder generalizado precisam ser melhor estudadas e controladas, uma vez que dos 19 estudos que apresentaram dados de generatividade, doze deles apresentaram dados parciais. Se, em conformidade com a afirmações anteriores, a generatividade é importante para a formação de meios mais eficientes e econômicos de ensino, esclarecer as variáveis que a controlam ou interferem poderia aumentar a frequência de dados totais na literatura da análise do comportamento e apresentar soluções relevantes para áreas educacionais e terapêuticas.

Além disso, a aplicabilidade deste procedimento em ambientes de menor controle e mais próximos do contexto natural de aprendizagem dos repertórios ensinados precisa ser mais extensivamente testada para o uso dessa tecnologia de ensino na resolução de problemas educacionais relacionados à leitura e à escrita.

\section{Referências}

Assis, G.J.A., Baptista, M.Q.G., \& Nunes, A.L. de M. (2009). Formação de sequências: aspectos conceituais, metodológicos e questões de pesquisa. Interação em Psicologia, 13, 215-227. Recuperado de http://revistas.ufpr.br/psicologia/article/viewFile/11960/11368

Assis, G.J.A, Calado, J.I.F., \& de Souza, S.R. (2016). Escolha de acordo com o modelo com resposta construída: uso de reforçadores condicionais específicos. Perspectivas em análise do comportamento, 7, 165-182. doi: 10.18761/pac.2015.025

Assis, G. J. A., Fonseca, A. C. G., \& Bandeira, T. M. (2014). Efeito do ensino da resposta por construção de sentenças sobre a leitura generalizada recombinativa. Comportamento em Foco, 4, 155-171. Recuperado de http://abpmc.org.br/ arquivos/publicacoes/1411068729e85fb38db. pdf

Benitez, P., \& Domeniconi, C. (2016). Use of a com- putadorized Reading and writing teaching program for families of students with intellectual disabilities. The Psychological Recorder, 66, 127138. doi:10.1007/s40732-015-0158-8

Birnie-Selwyn, B., Guerin, B. (1997). Teaching children to spell: decreasing consonant cluster errors by eliminating selective stimulus control. Journal of Applied Behavior Analysis, 30, 69-91. doi: 10.1901/jaba.1997.30-69

Calcagno, S., Dube, W. V., Galvão, O. F., \& Sidman, M. (1994). Emergence of conditional discriminations after constructed-response matching-to-sample training. The Psychological Record, $44,509-520$

Carroll, R.A., Joachim, B.T., St. Petter, C.C., \& Robinson, N. (2015). A comparison of error-correction procedures on skill acquisition during discrete-trial instruction. Journal of Applied Behavior Analysis, 48, 257-273. doi: 10.1002/jaba.205

Catania, A. C. (1999). Aprendizagem: Comportamento, linguagem e cognição. 4 ed. Porto Alegre, RS: ArtMed.

Debert, P., Matos, M.A., Andery, M. A. P.A. (2006). Discriminação condicional: definições, procedimentos e dados recentes. Revista Brasileira de Análise do Comportamento, 2, 37-52. DOI: http://dx.doi.org/10.18542/rebac.v2i1.801 de Paula, J.B.C., \& Haydu, V.B. (2010). Revisão Bibliográfica de Pesquisas Brasileiras sobre Equivalência de Estímulos. Psicologia: Teoria e Pesquisa, 26, 281-294. Recuperado de http:// dx.doi.org/10.1590/S0102-37722010000200010 de Rose, J. C. (1996). Controlling factors in conditional discriminations and tests of equivalence. In T. R. Zentall \& P. M. Smeets (Orgs.). Stimulus class formation in humans and animals. North Holland: Elsevier.

de Rose, J.C., de Sousa, D.G., \& Hanna, E.S. (1996). Teaching reading and spelling: exclusion and stimulus equivalence. Journal of Applied Behavior Analysis, 29, 451-469. doi: 10.1901/ jaba.1996.29-451

de Souza, D.G., \& de Rose, J.C. (2006). Desenvolvendo programas individualizados para ensino de leitura. Acta Comportamentalia, 14, 77-98. http://pepsic.bvsalud.org/pdf/actac/ v14n1/v14n1a04.pdf 
de Souza, D.G., de Rose, J.C., Faleiros, T.C., Bortoloti, R., Hanna, E.S., \& McIlvane, W.J. (2009). Teaching Generative Reading Via Recombination of Minimal Textual Units: A Legacy of Verbal Behavior to Children in Brazil. International Journal of Psychology and Psychological Therapy, 9, 19-44. Recuperado de https://www.ncbi.nlm.nih.gov/pmc/articles/ PMC2786216/

de Souza, S.R, Goyos, C., Silvares, E.F.M., \&Saunders, R.R. (2007) Emergence of Printing and Spelling Skills from Constructed-Response Matching-toSample Instruction (CRMTS). European Journal of Behavior Analysis, 8, 49-64. Recuperado de http://dx.doi.org/10.1080/1502 1149.2007.11434273

de Souza, S.R., \& Hubner, M. (2010). Efeitos de um jogo de tabuleiro educativo na aquisição de leitura e escrita. Acta comportamentalia, 18, 215242. Recuperado de http://pepsic.bvsalud.org/ pdf/actac/v18n2/a03.pdf

Domeniconi, C., de Rose, J.C., \& Huziwara, E.M. (2007). Equivalência de estímulos em participantes com síndrome de down: efeitos da utilização de palavras com diferenças múltiplas ou críticas e análise de controle restrito de estímulos. Revista Brasileira de Análise do Comportamento, 3, 47-63. doi: http://dx.doi. org/10.18542/rebac.v3i1.823

Dube, W. V., McDonald, S. J., Mcllvane, W. J., \& Mackay, H. A. (1991). Constructed-response matching to sample and spelling instruction. Journal of Applied Behavior Analysis, 24, 305317. doi: 10.1901/jaba.1991.24-305

Fonseca, A.C.G., Assis, G.J.A., \& de Souza, S.R. (2015). Efeito do ensino de sentenças sobre a leitura recombinativa com compreensão: procedimento de CRMTS. Revista Brasileira de Terapia Comportamental e Cognitiva, 17, 55-69. Recuperado de http://www.usp.br/rbtcc/index. php/RBTCC/article/view/815/449

Goulart, P. R. K., Delage, P. E. G. A., Rico, V. V., \& Brino, A. L. F. (2012). Aprendizagem. Em: Hübner, M. M. C., Moreira, M. B. (Orgs.). Temas clássicos da psicologia sob a ótica da análise do comportamento (pp. 20-41). Rio de Janeiro: Guanabara Koogan.

Greer, R. D. (2002). Designing teaching strategies: an applied behavior analysis systems approach. San Diego: Elsevier Academic Press.

Hanna, E. S., de Souza, D. G., de Rose, J. C., \& Fonseca, M. (2004). Effects of delayed constructed-response identity matching on spelling of dictated words. Journal of Applied Behavior Analysis, 37, 223-227. doi: 10.1901/ jaba.2004.37-223

Haydu, V.B., Zuanazzi, A.C., Assis, G.J.A., \& Kato, O.M. (2015). Ensino de Leitura de Sentenças: Contribuições da Análise do Comportamento. Psicologia: Teoria e Pesquisa, 31, 145-154. doi: 10.1590/0102-37722015021869145154

Lee-Vieira, A., Mayer, M. D., \& Cameron, M.J. (2006). Constructed-response spelling and literacy development: an application in an urban classroom. Behavioral Interventions, 21, 111122. doi: 10.1002/bin209

Mackay, H. A. (1985). Stimulus equivalence reading and in rudimentary spelling. Analysis and Intervention in Developmental Disabilities, 5, 373-387.

Mackay, H.A. (2013). Developing Syntactic Repertoires: Syntheses of Stimulus Classes, Sequences, and Contextual Control. European Journal of Behavior Analysis, 14, 69-85. Recuperado de http://dx.doi.org/10.1080/150 21149.2013.11434446

Mackay, H. A., \& Sidman, M. (1984). Teaching new behavior via equivalence relations. In: P. H. Brooks; R. Sperber \& C. McCauley (Eds.), Learning and cognition in the mentally retarded (pp. 493-513). Hillsdale, NJ: Erlbaum

Magalhães, P. G. S. \& Assis, G. J. A. (2011). Equivalência monetária em surdos. Temas em Psicologia, 19, 443-458. Recuperado de http:// pepsic.bvsalud.org/pdf/tp/v19n2/v19n2a08.pdf Martone, M.C.C., \& Santos-Carvalho, L.H.Z. (2012). Uma Revisão dos Artigos Publicados no Journal of Applied Behavior Analysis (JABA) sobre Comportamento Verbal e Autismo entre 2008 e 2012. Perspectivas em análise do comportamento, 3, 73-86. Recuperado de http://media. wix.com/ugd/89bfd6_007c08ad4e684cea80f35 7f535116915.pdf

Matos, M.A. (1999). Controle de estímulo condicional, formação de classes de equivalência e comportamentos cognitivos. Revista Brasileira 
de Terapia Comportamental e Cognitiva, 1, 159178. Recuperado de http://pepsic.bvsalud.org/ $\mathrm{pdf} / \mathrm{rbtcc} / \mathrm{v} 1 \mathrm{n} 2 / \mathrm{v} 1 \mathrm{n} 2 \mathrm{a} 06 . \mathrm{pdf}$

Matos, M. A., Avanzi, A. L, \& McIlvane, W. J. (2006). Rudimentary reading repertoires via stimulus equivalence and recombination of minimal verbal unit. The Analysis of Verbal Behavior, 22, 3-19. Recuperado de https://www. ncbi.nlm.nih.gov/pmc/articles/PMC2778457/

Mayfield, K. H., Glenn, I. M., \& Vollmer, T. R. (2008). Teaching Spelling Through Prompting and Review Procedures Using Computer-Based Instruction. Journal of Behavioral Education, 17, 303-312. Doi: 10.1007/s10864-008-9069-y

Reis, T. S., Postalli, L. M., \& de Souza, D. G. (2013). Teaching spelling as a route for reading and writing. Psychology \& Neuroscience, 6, 365-373. doi: 10.3922/j.psns.2013.3.14

Resende, A.A.C., Elias, N.C., \& Goyos, C. (2012). Transferência de funções ordinais através de classes de estímulos equivalentes em surdos. Acta Comportamentalia, 20, 317-326. http:// pepsic.bvsalud.org/pdf/actac/v20n3/a05.pdf

Rossit, R. A. S., \& Ferreira, P. R. S. (2003). Equivalência de Estímulos e o ensino de pré-requisitos monetários para pessoas com deficiência intelectual. Temas em Psicologia, 11, 97106. Recuperado de http://pepsic.bvsalud.org/ $\mathrm{pdf} / \mathrm{tp} / \mathrm{v} 11 \mathrm{n} 2 / \mathrm{v} 11 \mathrm{n} 2 \mathrm{a} 03 . \mathrm{pdf}$

Sampaio, A.A.S., de Azevedo, P.H.B., Cardoso, L.R.D., de Lima, C., Pereira, M.B.R., Andery, M.A.P.A (2008). Uma Introdução aos Delineamentos Experimentais de Sujeito Único. Interação em Psicologia, 12, 151164. http://revistas.ufpr.br/psicologia/article/ view/9537/9218

Santos, R. E. A., Assis, G. J. A., \& Borba, M. M. C. (2016). Ensino de Discriminações Condicionais de Sentenças Sobre a Emergência de Relações Sintáticas para Surdos. Perspectivas em análise do comportamento, 7, 86-100. doi: 10.18761/ pac. 2015.033

Santos, A.C.G., Simonassi, L.E., Rodrigues, C.F., \& Magri, M.R. (2014). Efeito do Treino de Composição (Cópia) na Aprendizagem do Conceito de Proporção. Psicologia: Teoria e Pesquisa, 30, 459-469. Recuperado de http:// www.scielo.br/pdf/ptp/v30n4/v30n4a11.pdf
Sella, A. C., Tenório, J.P., Bandini, C.S.M., \& Bandini, H.H.M. (2016). Games as a measure of Reading and writing generalization after computadorized teaching of reading skills. Psicologia: Reflexão e Crítica, 29, 1-12. doi: 10.1186/s41155-016-0039-3

Sidman, M., \& Tailby, W. (1982). Conditional discrimination vs. matching to sample: an expansion of the testing paradigm. Journal of the Experimental Analysis of Behavior, 37, 5-22. doi: 10.1901/jeab.1982.37-5

Skinner, B. F. (1938). The behavior of organisms: an experimental analysis. Oxford: AppletonCentury.

Skinner, B. F. (1992). Verbal behavior. Acton, Massachusetts: Copley (originalmente publicado em 1957).

Stromer, R., \& Mackay, H. A. (1992a). Delayed constructed-response identity matching improves the spelling performance of students with mental retardation. Journal of Behavioral Education, 2 139-156. doi: 10.1007/BF00947117

Stromer, R., \& Mackay, H. A. (1992b). Spelling and emergent picture-printed word relations established with delayed identity matching to complex samples. Journal of Applied Behavior Analysis, 25, 893-904. doi: 10.1901/jaba.1992.25-893

Stromer, R., \& Mackay, H.A (1993). Delayed Identity Matching to Complex Samples: Teaching Students With Mental Retardation Spelling and the Prerequisites for Equivalence Classes. Research in Developmental Disabilities, 14, 19-38. Recuperado de http://www.sciencedirect.com/science/article/pii/0891422293900033 Stromer, R., Mackay, H. A., Howell, S. R., McVay, A. A., \& Flusser, D. (1996). Teaching computer-based spelling to individuals with developmental and hearing disabilities: transfer of stimulus control to writing tasks. Journal of Applied Behavior Analysis, 29, 25-42. doi: 10.1901/ jaba.1996.29-25

Sudo, C. H., Soares, P. G., de Souza, S. R., \& Haydu, V. B. (2008). Equivalência de estímulos e uso de jogos para ensinar leitura e escrita. Revista Brasileira de Terapia Comportamental e Cognitiva, 2, 223 - 238. Recuperado de http:// www.usp.br/rbtcc/index.php/RBTCC/article/ view/228/181 
Sugasawara, H., \& Yamamoto, J. (2007). Computerbased teaching of word construction and reading in two students with developmental disabilities. Behavioral Interventions, 22, 263-277. doi: 10.1002/bin.248

Sugasawara, H., \& Yamamoto, J. (2009). Computerbased teaching of kanji construction and writing in a student with developmental disabilities. Behavioral Interventions, 24, 43-53. doi: 10.1002/bin. 271

Tanji, T., \& Noro, F. (2011). Matrix training for generative spelling in children with autism spectrum disorder. Behavioral Interventions, 26, 326-339. doi: 10.1002/bin.340

Vedora, J., \& Stromer, R. (2007). Computer-based spelling instruction for students with developmental disabilities. Research in Developmental Disabilities, 28, 489-505. doi: 10.1016/j. ridd.2006.06.006

Yamamoto, J., \& Miya, T. (1999). Acquisition and transfer of sentence construction in autistic students: analysis by computer-based teaching. Research in Developmental Retardation, 20 (5), 355-377. Recuperado de http://dx.doi. org/10.1016/S0891-4222(99)00017-7

Zanco, G., \& Moroz, M. (2015). Ensino de Leitura de Orações por meio de Discriminações Condicionais. Psicologia: Teoria e Pesquisa, 4, 509-517. doi: 10.1590/010237722015042363509517

\section{Informações do Artigo}

Histórico do artigo:

Submetido em: 07/10/2016

Primeira decisão editorial: 19/12/2016

Aceito em: 02/02/2017

Editor Associado: Lidia Maria Marson Postalli 